\title{
OSTEOTOMIA DA BASE DO I METATARSAL NO TRATAMENTO DO HÁLUX VALGO MODERADO E GRAVE: RESULTADOS APÓS SEGUIMENTO MÉDIO DE OITO ANOS
}

\author{
OSTEOTOMY OF THE TST METATARSAL BASE ON THE TREATMENT OF MODERATE TO \\ SEVERE HALLUX VALGUS AFTER MEAN FOLLOW-UP TIME OF 8 YEARS
}

Marco Túlio Costa', Roberto Zambelli de Almeida Pinto², Ricardo Cardenuto Ferreira', Minoru Alessandro Sakata', Gastão Guilherme Frizzo', Roberto Attílio Lima Santin ${ }^{3}$

\section{RESUMO}

Objetivos: Avaliar o resultado da correção do hálux valgo moderado ou grave com a osteotomia da base do I metatarsal, associada à liberação distal de partes moles e capsuloplastia medial. Métodos: Foram avaliados 13 pacientes (15 pés), submetidos à cirurgia para correção de hálux valgo moderado ou grave. O tempo médio de seguimento foi de 102 meses, 12 pacientes eram do sexo feminino e um do masculino, com média de idade, no momento da cirurgia, de 49 anos. Os pacientes convocados foram entrevistados segundo questionário desenvolvido no serviço, avaliados clinicamente conforme o escore de AOFAS e submetidos a exames radiográficos para comparação com os pré-operatórios. Resultados: A média da pontuação da escala da AOFAS obtida na avaliação final foi de 82 pontos. Nove dos 15 pés $(60 \%)$ apresentaram alguma complicação tardia, sendo quatro $(27 \%)$ deformidades em varo, três $(20 \%)$ recidivas; dois pacientes $(13 \%)$ com queixa de dor sem deformidade associada. $\mathrm{Na}$ articulação metatarsofalângica do hálux a perda do movimento foi de $41^{\circ}(57 \%)$; o movimento em dorsiflexão foi o mais afetado, com perda de $37^{\circ}$ em média (60\%). A amplitude de movimento do lado contralateral não operado foi utilizada como grupo controle. Observou-se progressão da artrose na avaliação radiográfica final. Em todos os casos, houve encurtamento e elevação do primeiro metatarsal, porém não foi possível correlacionar o encurtamento e elevação com metatarsalgia, calosidade plantar ou menor pontuação na escala AOFAS na avaliação final. Conclusões: A técnica de osteotomia da base e realinhamento distal de partes moles, utilizada para correção de hálux valgo moderado a grave, mostrou elevado índice de complicações tardias. Devido ao elevado número dessas complicações, acreditamos que a eleição dessa técnica para correção do hálux valgo deve ser considerada com cautela.

Descritores - Hallux valgus; Articulação metatarsofalângica; Ossos do metatarso; Cirurgia; Osteotomia; Seguimentos

\section{ABSTRACT}

Objective: To assess the result of the treatment of moderate to severe hallux valgus with osteotomy of the first metatarsal base associated to distal release of soft parts and medial capsuloplasty. Methods: 13 patients were assessed (15 feet) submitted to surgical treatment of hallux valgus moderate to severe. The mean follow-up time was 102 months; there were 12 female and 1 male patients, with mean age at the time of surgery of 49 years. The patients enrolled were interviewed according to the questionnaire developed by our service, clinically examined according to the AOFAS scale and submitted to X-ray tests for comparing the results with baseline images. Results: The mean score of the AOFAS scale obtained at the final assessment was 82 points. Nine of the 15 feet (60\%) showed some late complications, with four (27\%) varus deformities, three (20\%) recurrences; two patients (13\%) presenting with pain complaints with no associated deformity. In the hallux metatarsophalangeal joint, movement loss was $41^{\circ}(57 \%)$; dorsiflexion movement was mostly affected, with a mean loss of $37^{\circ}(60 \%)$. The range of motion on the contralateral intact side served as control group. Arthrosis progression was seen on the final X-ray evaluation. In all cases, shortening and lifting of the first metatarsal were noticed; however, we couldn't correlate the shortening and lifting with metatarsalgia, plant callosity or lower scoring on the AOFAS scale at the final evaluation. Conclusions: The osteotomy technique by the basis and with distal realignment of soft parts employed in the treatment of moderate to severe hallux valgus showed a high rate of late complications. Due to the high number of complications, we believe that adopting this technique for correcting hallux valgus deformities should be carefully considered.

Keywords - Hallux valgus; Metatarsophalangeal joint; Metatarsal bones; Surgery; Osteotomy; Follow-up studies

1 - Médico Assistente do Grupo do Pé e Tornozelo da Santa Casa de Misericórdia de São Paulo, SP, Brasil.

2 - Médico Ex-estagiário do Grupo do Pé e Tornozelo da Santa Casa de Misericórdia de São Paulo, SP, Brasil.

3 - Médico Chefe do Grupo do Pé e Tornozelo da Santa Casa de Misericórdia de São Paulo, SP, Brasil.

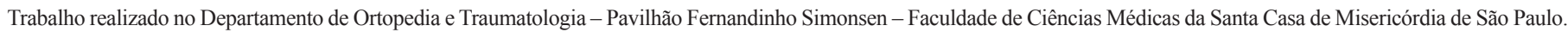
Correspondência: Rua João Moura, 2.245 - São Paulo, SP. CEP: 05412-004., E-mail: tuliom@uol.com.br 


\section{INTRODUÇÃO}

As osteotomias proximais do I metatarsal, associadas à liberação de partes moles distal, tem sido descritas como opção de tratamento para hálux valgo considerado moderado e grave ${ }^{(1-3)}$. Alguns autores enfatizam a capacidade da correção angular e posição dos sesamoides como a principal vantagem desse tipo de abordagem cirúrgica $^{(1,4,5)}$. No entanto, outros documentaram dificuldades de manutenção da anatomia do I metatarsal, assim como dificuldade na fixação da osteotomia, o que poderia levar a consolidação viciosa com elevação do I metatarsal ${ }^{(6-9)}$. Além disso, uma tendência de alteração nos padrões de pressão plantar nos pacientes submetidos à osteotomia proximal do I metatarsal, levando a metatarsalgias de transferência, seria uma possível complicação do procedimento ${ }^{(6,8)}$, devido ao possível encurtamento e elevação do I metatarsal.

A incidência de complicações nesse tipo de abordagem é relativamente elevada, principalmente hálux varo e recidiva da deformidade ${ }^{(5,8)}$. Outra complicação bastante notada na prática clínica, mas pouco documentada, é a restrição de movimento da articulação metatarsofalângica do primeiro raio ${ }^{(1)}$.

O objetivo deste estudo é avaliar o resultado da correção do hálux valgo moderado ou grave com a osteotomia da base do I metatarsal, associada à liberação de partes moles distal e capsuloplastia medial.

Nossa hipótese é de que essa combinação de procedimentos levaria a alta incidência de elevação do primeiro raio, com consequente aumento das lesões por transferência da carga para os raios laterais, culminado com deterioração clínico-funcional dos pacientes em longo prazo.

\section{MÉTODOS}

Foram incluídos neste trabalho 13 pacientes (15 pés) com diagnóstico de hálux valgo sintomático, moderado ou grave, segundo a classificação de Coughlin ${ }^{(3)}$, submetidos à osteotomia da base do I metatarsal, liberação de partes moles distal e capsuloplastia medial, operados pelo Grupo do Pé e Tornozelo da Santa Casa de Misericórdia de São Paulo. Consideramos critérios de exclusão os pacientes com diagnóstico de artrite reumatoide e hálux valgo juvenil, além daqueles submetidos a cirurgias prévias no mesmo pé. Segundo os critérios definidos por Coughlin ${ }^{(3)}$, são consideradas deformidades moderadas aquelas com ângulo intermetatarsal entre $11^{\circ}$ e $16^{\circ}$, metatarsofalangiano, o entre $20^{\circ}$ e $40^{\circ}$, e graves aquelas com ângulo intermetatarsal maior que $16^{\circ}$ e o metatarsofalangiano maior que $40^{\circ}$.
O tempo médio de seguimento foi 102 meses (com variação de 26 a 202 meses). Doze pacientes eram do sexo feminino e um do masculino. A média de idade, no momento da cirurgia, era de 49 anos (variando de 19 a 76 anos).

A osteotomia utilizada na correção da deformidade foi a do tipo cunha lateral de subtração em nove pés (60\%), tipo chevron proximal em cinco pés (33\%) e cupuliforme em um pé (7\%). A fixação com fios foi utilizada em 12 pés (80\%) e com parafusos em três pés (20\%) (Tabela 1$)$.

Foram realizadas, concomitantemente, as seguintes cirurgias: realinhamento da articulação metatarsofalângica do III dedo em dois pés, para tratamento de subluxação dorsal da falange proximal; artrodese da articulação IFP do II dedo em quatro pés, para correção de deformidade em garra rígida; osteotomia de deslizamento medial do calcâneo em um pé, para tratamento de deformidade em valgo excessivo do retropé; transferência do tendão do flexor longo do II dedo para o complexo extensor em um pé, para correção de deformidade em garra flexível; e alongamento do tendão extensor longo do hálux em um pé.

\section{Avaliação clínico-funcional}

Os pacientes foram questionados quanto à presença ou não de dor no antepé, deformidade, limitação na vida diária, capacidade de marcha e distância máxima percorrida, além da satisfação com o resultado final do procedimento cirúrgico. A escala funcional para a articulação metatarsofalângica do hálux da $\mathrm{AOFAS}^{(}$(American Orthopaedic Foot and Ankle Society) ${ }^{(10)}$ foi utilizada para mesurar quantitativamente os resultados pós-operatórios.

No exame clínico anotamos o alinhamento e posição do pé durante apoio, presença ou não de deformidades no hálux (valgo, varo, flexo ou extenso), alterações no demais dedos (garra ou martelo). A seguir, com o paciente sentado na mesa de exame, investigamos a presença de calosidades ou alterações sensitivas (áreas de hipoestesia ou presença de neuromas) e a amplitude de movimento das articulações do tornozelo, subtalar, mediopé, metatarsofalângica (MTF) do hálux. Como não dispúnhamos da mobilidade articular no exame pré-operatório de todos os pacientes, utilizamos como grupo controle os pés não operados dos pacientes. Foram excluídos do grupo controle os pacientes submetidos à cirurgia nos dois pés.

\section{Avaliação radiográfica}

Os exames radiográficos pré-operatórios do antepé foram realizados com carga na projeções dorsoplantar e perfil. Essas radiografias foram comparadas com as primeiras pós-operatórias realizadas com carga cerca de 
12 semanas após a cirurgia e também com as feitas no momento da última avaliação clínico funcional.

Os parâmetros radiográficos utilizados para avaliação e comparação foram os seguintes:

Ângulo intermetatarsal I-II ${ }^{(3)}$ (AIM I-II): ângulo formado entre as linhas que bisseccionam as diáfises do I e II metatarsal (MTT);

Ângulo de valgismo do hálux ${ }^{(3)}$ (AHV): ângulo formado entre as linhas que bisseccionam a diáfise da falange proximal e a do I metatarsal;

Posição dos sesamoides, de acordo com a classificação de Smith e Reynolds ${ }^{(7)}$, que utiliza como referência a posição do sesamoide tibial em relação ao eixo longitudinal do I MTT, onde o grau 0 é normal, ou seja, o sesamoide tibial está medial ao eixo; no grau 1 ocorre sobreposição menor de 50\%; no grau 2, sobreposição maior 50\%; e no grau 3, desvio lateral;

Ângulo articular metatarsal distal ${ }^{(3)}$ (DMAA): determina-se o ponto mais lateral e o ponto mais medial da superfície articular distal do I MTT; traça-se uma linha conectando os dois pontos. A seguir, traça-se uma linha no eixo do I MTT e mede-se o ângulo entre as duas linhas;

Artrose da articulação metatarsofalângica (MF) do hálux, classificação de Hattrup e Johnson ${ }^{(1)}$, sendo o grau 0 considerado normal; grau 1, caracterizado pela presença de osteófitos leves ou moderados; o grau 2, osteófitos moderados, com diminuição do espaço articular e esclerose; e o grau 3, osteófitos associados à diminuição importante do espaço articular, com ou sem a presença de cistos. Para esse dado, apenas utilizamos os exames radiográficos pré-operatórios e pós-operatórios finais;

Elevação do I metatarsal ${ }^{(6)}$ : traça-se uma linha de referência entre o ponto mais plantar da tuberosidade do calcâneo e o ponto mais plantar da cabeça do V MTT. A seguir, traça-se uma perpendicular à anterior até o ponto mais plantar da cabeça do I MTT (d). Essa distancia "d" equivale à elevação do I MTT; (Figura 1).

Encurtamento do I metatarsal ${ }^{(11)}$ : traça-se uma linha no longo eixo do I MTT do ponto mais distal ao ponto mais proximal (A); o mesmo se faz para o II MTT (B). A mensuração do encurtamento faz-se pela diferença entre A e B. Valores negativos correspondem a index minus e valores positivos, a index plus (Figura 2).

Utilizamos, para avaliação estatística dos dados obtidos, os testes $t$, pareados ou independentes, para os dados que se apresentavam dentro da normalidade, e

Tabela 1 - Distribuição dos pacientes avaliados com relação a idade (em anos), sexo, tipo de osteotomia e fixação, complicações precoces e tardias, dor residual, amplitude de movimento na MTF do hálux, escala da AOFAS para MTF do hálux e satisfação pessoal com o resultado obtido.

\begin{tabular}{|c|c|c|c|c|c|c|c|c|c|c|c|c|}
\hline \multirow[b]{2}{*}{ Caso } & \multirow[b]{2}{*}{ Idade } & \multirow[b]{2}{*}{ Sexo } & \multirow[b]{2}{*}{ Deformidade } & \multirow[b]{2}{*}{$\begin{array}{c}\text { Tipo de } \\
\text { osteotomia }\end{array}$} & \multirow[b]{2}{*}{ Fixação } & \multirow[b]{2}{*}{$\begin{array}{l}\text { Comp. } \\
\text { precoces }\end{array}$} & \multirow[b]{2}{*}{$\begin{array}{l}\text { Comp. } \\
\text { tardias }\end{array}$} & \multirow[b]{2}{*}{ Dor residual } & \multicolumn{2}{|c|}{$\begin{array}{c}\text { Mobilidade MTF } \\
\text { hálux }\end{array}$} & \multirow[b]{2}{*}{ AOFAS } & \multirow[b]{2}{*}{ Satisfação } \\
\hline & & & & & & & & & $\begin{array}{l}\text { Flexão } \\
\text { plantar }\end{array}$ & Dorsiflexão & & \\
\hline 1 & 48 & $\mathrm{~F}$ & Grave & Cunha lateral & 1 fio $K$ & & Hálux Varo & \begin{tabular}{|c|} 
Moderada - \\
cabeça II e III MTT \\
\end{tabular} & 20 & 20 & 52 & Insatisfeita \\
\hline 2 & 44 & $\mathrm{~F}$ & Grave & Cupuliforme & 1 fio $K$ & & & $\begin{array}{c}\text { Leve - cabeça II e } \\
\text { III MTT }\end{array}$ & 30 & 30 & 85 & Satisfeita \\
\hline 3 & 53 & $\mathrm{~F}$ & Grave & Cunha lateral & 1 fio $K$ & & & Não & 0 & 20 & 73 & $\begin{array}{c}\text { Satisfeito com } \\
\text { mínimas restrições }\end{array}$ \\
\hline 4 & 19 & $\mathrm{~F}$ & Grave & Cunha lateral & 2 fios $K$ & & & Não & 30 & 30 & 95 & Satisfeita \\
\hline 5 & 76 & $\mathrm{~F}$ & Grave & Cunha lateral & 1 fio $K$ & & & Não & 0 & 30 & 95 & Satisfeito \\
\hline 6 & 57 & $\mathrm{~F}$ & Moderada & Cunha lateral & \begin{tabular}{|c|}
1 paraf. cortical \\
$3,5 \mathrm{~mm}$
\end{tabular} & & Hálux varo & $\begin{array}{c}\text { Leve - cabeça II, } \\
\text { III e V MTT }\end{array}$ & 45 & 35 & 75 & Insatisfeita \\
\hline $6^{\prime}$ & & $\mathrm{F}$ & Grave & Cunha lateral & $\begin{array}{c}1 \text { paraf. cortical } \\
3,5 \mathrm{~mm}\end{array}$ & & Hálux varo & Não & 50 & 35 & 75 & Insatisfeita \\
\hline 7 & 63 & $\mathrm{M}$ & Grave & Cunha lateral & 2 fios $K$ & & & Não & 20 & 10 & 95 & Satisfeito \\
\hline 8 & 44 & $\mathrm{~F}$ & Moderada & Cunha lateral & $\begin{array}{c}1 \text { paraf. } \\
\text { canulado } 4,0 \mathrm{~mm}\end{array}$ & & Hálux varo & \begin{tabular}{|c|} 
Moderada - \\
cabeça II e III MTT \\
\end{tabular} & 20 & 20 & 45 & $\begin{array}{c}\text { Satisfeita com } \\
\text { maiores restrições }\end{array}$ \\
\hline 9 & 59 & $\mathrm{~F}$ & Grave & Chevron & 2 fios $K$ & & & Não & 30 & 10 & 95 & Satisfeita \\
\hline 10 & 25 & $\mathrm{~F}$ & Grave & Chevron & 2 fios $K$ & $\begin{array}{l}\text { Infecção } \\
\text { superficial }\end{array}$ & Recidiva & Leve - MTF hálux & 30 & 50 & 73 & Insatisfeito \\
\hline 11 & 59 & $\mathrm{~F}$ & Grave & Chevron & 1 fio $K$ & & Recidiva & Não & 20 & 20 & 90 & $\begin{array}{c}\text { Satisfeito com } \\
\text { maiores restrições }\end{array}$ \\
\hline 12 & 68 & $\mathrm{~F}$ & Grave & Cunha lateral & 2 fios $K$ & & & Não & 45 & 30 & 100 & Satisfeito \\
\hline 13 & 25 & $F$ & Grave & Chevron & 2 fios $K$ & & Recidiva & Não & 60 & 15 & 88 & $\begin{array}{c}\text { Satisfeito com } \\
\text { mínimas restrições }\end{array}$ \\
\hline $13^{\prime}$ & & $\mathrm{F}$ & Grave & Chevron & 3 fios $K$ & & & Não & 60 & 15 & 95 & $\begin{array}{c}\text { Satisfeito com } \\
\text { mínimas restrições }\end{array}$ \\
\hline
\end{tabular}

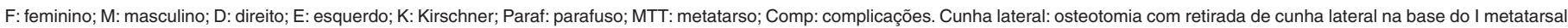
Fonte: SAME da Santa Casa de Misericórdia de São Paulo 

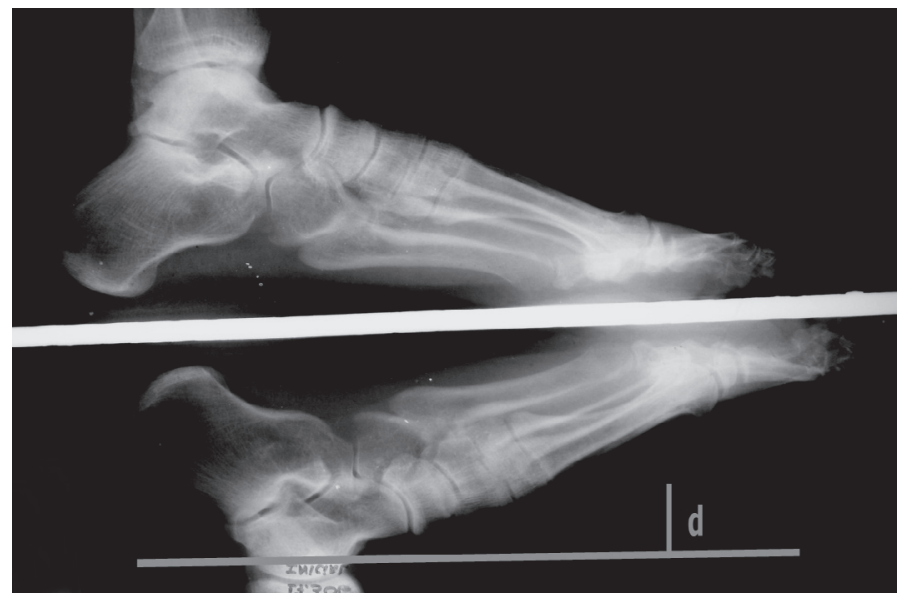

Figura 1 - Imagem radiográfica ilustrando o método da mensuração da elevação do I MTT em relação ao solo.

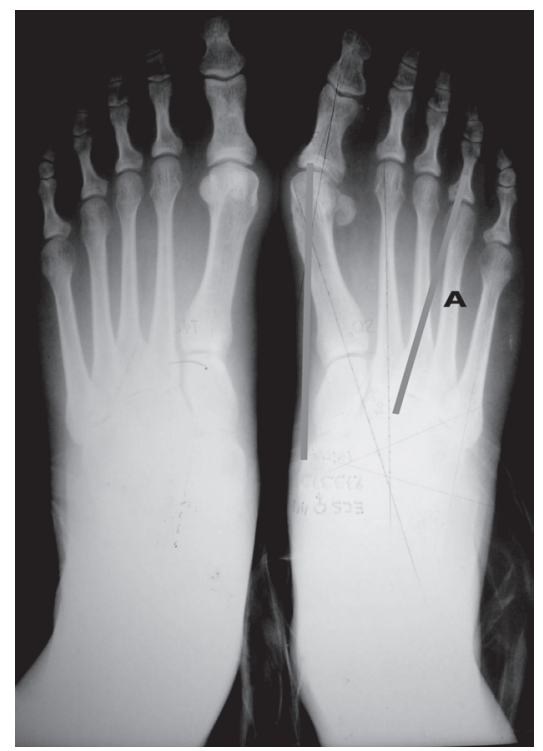

Figura 2 - Imagem radiográfica ilustrando a mensuração do encurtamento do I MTT em relação ao II, onde o valor é expresso pela diferença A-B.

os testes de Mann-Whitney e Wilcoxon para aqueles que não foram aceitos pela normalidade. Consideramos valores significativos do ponto de vista estatístico aqueles onde $p<0,05$. Para estabelecer correlação entre encurtamento, elevação e a avaliação pela AOFAS, utilizamos o coeficiente de correlação (r), cuja análise se dá a partir do quadro 1 .

Quadro 1 - Parâmetros de análise do coeficiente de correlação (r)

\begin{tabular}{|c|c|}
\hline$<0,25$ & Não há correlação ou a correlação é fraca \\
\hline $0,25-0,5$ & Pequena correlação \\
\hline $0,5-0,75$ & Correlação moderada a boa \\
\hline$>0,75$ & Correlação boa a ótima \\
\hline Valores positivos: correlação direta & Valores negativos: correlação indireta \\
\hline
\end{tabular}

\section{RESULTADOS}

Todos os pacientes foram avaliados pelo mesmo examinador, que não participou em nenhum momento do tratamento realizado. Quanto ao escore da AOFAS, o valor médio obtido na avaliação final foi de 82 pontos (variação de 45 a 100 pontos).

Foi observada infecção superficial na ferida operatória em um pé, que evoluiu com recidiva precoce da deformidade. Nesse caso, foi realizada osteotomia tipo chevron proximal, fixada com fios de Kirschner de 2,0mm. Observamos artrose precoce da articulação metatarsofalângica em um pé, tratado com artrodese. Como complicações tardias, em quatro pés (três pacientes), notamos deformidade em varo sintomática do hálux. Todos esses casos haviam sido submetidos a osteotomia com retirada de cunha lateral; em três, foi realizada a fixação com parafusos. Apesar de os três pacientes (quatro pés) queixarem-se da deformidade, em apenas dois pés, foi realizada uma nova cirurgia para correção (artrodese da metatarsofalângica do hálux). A recidiva da deformidade ocorreu em três pacientes (três pés) que haviam sido submetidos à osteotomia tipo chevron proximal; no entanto, recusaram novo procedimento cirúrgico. Não foi observado retardo de consolidação ou pseudartrose.

Notamos, ao exame clínico, dor na face medial da articulação MTF do hálux em cinco pacientes (cinco pés); em três destes, havia deformidade em varo do hálux. A dor na MTF do hálux não esteve relacionada com a recidiva da deformidade em valgo. Três dos cinco pacientes com dor haviam sido submetidos a osteotomia com retirada de cunha lateral da base do I metatarsal. Com relação a calosidades plantares, estavam presentes em quatro pés, localizadas na região da cabeça do II e/ou III metatarsal. No entanto, somente duas eram sintomáticas. Na tabela 1 estão os dados gerais dos pacientes avaliados.

Avaliando a amplitude de movimento das articulações do pé e tornozelo, não observamos alterações significativas com relação ao tornozelo, articulação subtalar e à mobilidade do mediopé (pronação/supinação, adução/abdução). Na articulação MTF do hálux, em 13 dos 15 pés, havia perda de movimento, que foi em média de $41^{\circ}(57 \%$ de perda em relação ao grupo controle). $\mathrm{O}$ movimento em dorsiflexão foi o mais afetado, com perda de $37^{\circ} \mathrm{em}$ média (60\% de perda em relação ao grupo controle).

Dos 13 pacientes avaliados, apenas seis relataram estar completamente satisfeitos com o resultado do tratamento na avaliação final. Dois (três pés) referiram estar satisfeitos, porém, com mínima restrição, e outros dois, 
satisfeitos com maiores restrições. Três pacientes (quatro pés) encontravam-se insatisfeitos com o resultado final (dois destes com hálux varo e outro com recidiva da deformidade) (Tabela 1).

Os resultados com relação à avaliação radiográfica encontram-se na tabela 2. Quando realizada a análise estatística dos valores obtidos, observamos que apenas o DMAA não sofreu alteração.

Comparamos os resultados clínico-funcionais segundo a escala AOFAS para articulação MTF do hálux com o encurtamento e a elevação observada no exame radiográfico (Tabela 3). No entanto, não houve correlação estatística entre essas variáveis (Tabela 4).

Quando comparamos a presença ou não de dor na articulação MTF do hálux na avaliação pós-operatória final com a perda de movimento em dorsiflexão, também não observamos correlação estatística.

Observamos encurtamento e elevação do primeiro metatarsal em todos os casos. No entanto, não houve significância estatística na correlação entre a elevação e o encurtamento do primeiro metatarsal com a presença de calosidades plantares ou com menor pontuação na escala da AOFAS para articulação MTF do hálux

\section{DISCUSSÃO}

Osteotomias da base do I metatarsal, sejam elas em cunha de base lateral ${ }^{(8)}$, cunha de adição medial ${ }^{(12,13)}$, chevron proximal $^{(9,13)}$ ou cupuliforme ${ }^{(1,2,7,14)}$, sempre associadas a procedimentos de realinhamento distal com liberação da cápsula lateral e do tendão conjunto, são descritas para correção do hálux valgo moderado a grave. No entanto, alguns autores notaram que o emprego dessa técnica pode levar a elevação e/ou encurtamento do I metatarsal ${ }^{(4-6,14)}$, com consequente deterioração dos resultados clínico-funcionais em longo prazo, devido ao surgimento de calosidades e metatarsalgia de transferência. Na literatura nacional, não encontramos nenhum seguimento equivalente (média de oito anos) nos casos

Tabela 2 - Avaliação radiográfica dos pacientes com diagnóstico de hálux valgo tratados com a técnica de osteotomia proximal do I MTT e liberação de partes moles distal.

\begin{tabular}{|c|c|c|c|c|}
\hline & Pré-op. (média) & Pós-op. Inicial (média) & Pós-op. Final (média) & \\
\hline AHV & $35^{\circ}(18-47)$ & $14^{\circ}(-2-36)$ & $90(-40-46)$ & $p<0,0001^{* *}$ \\
\hline AIM I-II & $17^{\circ}(15-22)$ & $90(0-20)$ & $8^{\circ}(0-20)$ & $p<0,0001^{* \star}$ \\
\hline DMAA & $10^{\circ}(4-20)$ & $13^{\circ}(8-22)$ & $11^{\circ}(4-22)$ & $p=0,47$ \\
\hline Artrose $^{*}$ & $1(0-2)$ & & $2(1-3)$ & $p<0,0001^{* \star}$ \\
\hline Sesamoides* & $4(3-4)$ & & $2(1-4)$ & $p=0,0001^{* \star}$ \\
\hline Elevação & $0,77 \mathrm{~cm}(0-1,3)$ & $1,31 \mathrm{~cm}(0-2,3)$ & $1,30 \mathrm{~cm}(0,8-2,0)$ & $p<0,0001^{* \star}$ \\
\hline Encurtamento & $-1,29 \mathrm{~cm}(1,0-1,7)$ & $-1,70 \mathrm{~cm}(1,1-2,2)$ & $-1,73 \mathrm{~cm}(0,9-2,3)$ & $p<0,0001^{* \star}$ \\
\hline
\end{tabular}

AHV: ângulo de valgismo do hálux; AIM I-II: ângulo intermetatarsal I-II MTT; DMAA: ângulo articular metatarsal distal.

*: valor expresso pela mediana dos valores obtidos. Avaliação estatística é relativa aos dados pré-operatórios e pós-operatórios finais. ${ }^{* \star}$ Estatisticamente significativo ( $\left.\mathrm{p}<0,05\right)$.

Fonte: SAME da Santa Casa de Misericórdia de São Paulo.

Tabela 3 - Distribuição dos pacientes com relação ao tipo de osteotomia, escala AOFAS para MTF hálux, encurtamento e elevação do I metatarso, valores angulares pré e pós-operatórios do ângulo de hálux valgo e do ângulo entre o I metatarso e o II metatarso e da classificação da artrose na radiografia inicial e final segundo Harttrup e Johnson.

\begin{tabular}{|c|c|c|c|c|c|c|c|c|c|c|}
\hline $\begin{array}{c}\text { R.G. Grupo } \\
\text { Pé }\end{array}$ & $\begin{array}{c}\text { Tipo de } \\
\text { osteotomia }\end{array}$ & AOFAS & $\begin{array}{c}\text { Encurtamento } \\
(\mathrm{cm})\end{array}$ & Elevação (cm) & HV pré & HV final & IMTT 1-2 pré & IMTT 1-2 final & $\begin{array}{c}\text { Artrose MF } \\
\text { hálux inicial }\end{array}$ & $\begin{array}{l}\text { Artrose MF } \\
\text { hálux final }\end{array}$ \\
\hline 1 & Cunha lateral D & 52 & 0,60 & 1,00 & 18,00 & $-42,00$ & 18,00 & 0,00 & 1,00 & 2,00 \\
\hline 2 & Cupuliforme D & 85 & 0,70 & 0,60 & 34,00 & 18,00 & 16,00 & 10,00 & 1,00 & 2,00 \\
\hline 3 & Cunha lateral & 73 & 0,50 & 1,00 & 37,00 & 16,00 & 17,00 & 8,00 & 0,00 & 1,00 \\
\hline 4 & Cunha lateral E & 95 & 0,20 & 0,40 & 40,00 & 14,00 & 17,00 & 6,00 & 0,00 & 3,00 \\
\hline 5 & Cunha lateral D & 95 & 0,30 & 0,70 & 45,00 & 28,00 & 16,00 & 6,00 & 1,00 & 1,00 \\
\hline 6 & Cunha lateral D & 75 & 0,30 & 0,30 & 25,00 & 5,00 & 15,00 & 8,00 & 1,00 & 1,00 \\
\hline 6 & Cunha lateral E & 75 & 0,40 & 1,10 & 32,00 & $-4,00$ & 17,00 & 7,00 & 2,00 & 3,00 \\
\hline 7 & Cunha lateral E & 95 & 0,20 & 0,20 & 47,00 & 12,00 & 22,00 & 6,00 & 2,00 & 2,00 \\
\hline 8 & Cunha lateral D & 45 & 0,30 & 0,40 & 33,00 & $-20,00$ & 16,00 & 6,00 & 2,00 & 2,00 \\
\hline 9 & Chevron proximal D & 95 & 0,30 & 0,60 & 30,00 & 18,00 & 15,00 & 12,00 & 1,00 & 1,00 \\
\hline 10 & Chevron proximal E & 73 & 0,70 & 0,10 & 40,00 & 46,00 & 18,00 & 20,00 & 0,00 & 2,00 \\
\hline 11 & Chevron proximal D & 90 & 0,30 & 0,60 & 44,00 & 20,00 & 16,00 & 10,00 & 1,00 & 2,00 \\
\hline 12 & Cunha lateral D & 100 & 0,60 & 0,80 & 32,00 & 7,00 & 17,00 & 9,00 & 1,00 & 2,00 \\
\hline 13 & Chevron proximal D & 88 & 0,70 & 0,10 & 28,00 & 32,00 & 16,00 & 18,00 & 0,00 & 1,00 \\
\hline $13^{\prime}$ & Chevron proximal E & 95 & 0,80 & 0,20 & 30,00 & 6,00 & 16,00 & 6,00 & 0,00 & 1,00 \\
\hline
\end{tabular}

D: direito; E: esquerdo; HV: ângulo de hálux valgo; MF: metatarsofalangiana. Valores negativos do ângulo de hálux valgo expressam deformidade em varo

Fonte: SAME da Santa Casa de Misericórdia de São Paulo. 
Tabela 4 - Dados relativos ao encurtamento e à elevação do I raio em relação ao segundo raio dos pacientes com diagnóstico de hálux valgo tratados com a técnica de osteotomia proximal do I MTT.

\begin{tabular}{|c|c|c|c|}
\hline Nome & AOFAS (pontos) & Encurtamento (cm) & Elevação $(\mathrm{cm})$ \\
\hline ANB & 52 & 0,60 & 1,00 \\
\hline EC & 85 & 0,70 & 0,60 \\
\hline IR & 73 & 0,50 & 1,00 \\
\hline SLD & 95 & 0,20 & 0,40 \\
\hline MMD & 95 & 0,30 & 0,70 \\
\hline EJJ & 75 & 0,30 & 0,30 \\
\hline EJJ" & 75 & 0,40 & 1,10 \\
\hline NDF & 95 & 0,20 & 0,20 \\
\hline TPM & 45 & 0,30 & 0,40 \\
\hline JRSF & 95 & 0,30 & 0,60 \\
\hline SRO & 73 & 0,70 & 0,10 \\
\hline SIS & 90 & 0,30 & 0,60 \\
\hline ANS & 100 & 0,60 & 0,80 \\
\hline MCAG & 88 & 0,70 & 0,10 \\
\hline MCAG" & 95 & 0,80 & 0,20 \\
\hline \multicolumn{4}{|c|}{ Coeficiente de correlação (r) Elevação $\times$ AOFAS $=-0,0495$} \\
\hline \multicolumn{4}{|c|}{ Coeficiente de correlação ( $r$ ) Encurtamento x AOFAS = -0,2097 } \\
\hline
\end{tabular}

AOFAS: escore da American Orthopedic Foot and Ankle Society para articulação metatarsofalangiana do hálux.

Fonte: SAME da Santa Casa de Misericórdia de São Paulo.

de osteotomia da base para a correção de deformidades em hálux valgo moderadas a grave.

Com tempo de seguimento médio de oito anos, a média do escore de AOFAS nos pacientes avaliados foi de 82 pontos (variação de 45 a 100). Os quesitos que contribuíram para a maior perda de pontos foram mobilidade da articulação MTF do hálux (13 pés dos 15 pés tinham limitação de movimento) e dor (cinco dos 15 pés tinham dor na avaliação final). Baykal et $a l^{(15)}$ observaram melhora de 54,4 para 95,4 pontos e $93,8 \%$ dos pés sem queixas álgicas, no entanto, em seguimento médio de 28 meses. Okuda et al ${ }^{(1)}$ demonstraram resultados semelhantes, com AOFAS final médio de 92 pontos, mas também com seguimento máximo de três anos, inferior ao tempo observado em nosso estudo. Já Brodsky et al ${ }^{(4)}$ encontraram AOFAS final médio de 86,4 pontos (variação de 71 a 100), em estudo em que foram acompanhados 32 pacientes por tempo médio de 29 meses. Apesar de a pontuação, segundo a escala da AOFAS, relatada por esses autores serem superiores à pontuação dos pacientes avaliados neste trabalho, o tempo de seguimento é também menor. Isso nos permite questionar se o resultado clínico funcional, medido pela escala AOFAS, não sofre deterioração com o aumento do tempo de seguimento.

A restrição de movimento na MTF do hálux ocorreu principalmente na dorsiflexão, com limitação de mais de $50 \%$ da mobilidade ( $37^{\circ} \mathrm{em}$ média). Okuda et al ${ }^{(2)}$ encontraram perda de apenas $7^{\circ}$ quanto à dorsiflexão e de $15^{\circ}$ de flexão plantar, em seguimento médio de 48 meses, utilizando medidas pré e pós-operatórias como parâmetro. Em nosso trabalho, utilizamos o pé contralateral como parâmetro de controle. Okuda et $a l^{(1)}$, em seguimento máximo de três anos, relataram perda de $21^{\circ}$ em toda a excursão de movimento da MTF do hálux, também utilizando como parâmetro a avaliação pré-operatória. Em nossa opinião, o tensionamento excessivo da cápsula articular, a má redução da articulação e a presença de artrose podem ser causas dessa restrição de movimento. Observamos que a maior perda da mobilidade articular ocorreu em dorsiflexão. A elevação do I metatarsal, ou seja, a angulação dorsal no local da osteotomia, observada em todos os nossos pacientes, também pode ser causa dessa restrição de movimento.

Alguns autores ressaltaram a capacidade de correção dos ângulos AHV, o AIM I-II e da posição dos sesamoides com a osteotomia proximal e o procedimento distal de partes moles ${ }^{(1-4,7,8)}$. No entanto, devemos considerar que o desvio em varo do hálux, complicação observada em quatro pacientes (27\%) em nosso trabalho, e demonstrada com valores angulares negativos, potencializa o poder de correção dessa técnica. Nos pacientes que evoluíram com varismo na MTF do hálux, o valor médio do AHV foi de $-15,3^{\circ}$. Quando excluímos esses pacientes do cálculo da média do AHV final, o valor da média desse ângulo foi de $19,7^{\circ}$, o que demonstra a não correção total da deformidade. Em relação ao AIM I-II, nos pacientes com hálux varo, a média foi de $5,3^{\circ}$, e nos demais, $10,1^{\circ}$. Portanto, quando descartamos os casos que evoluíram com deformidade em varo da MTF do hálux, podemos notar que a capacidade dessa técnica não é tão grande como se observara inicialmente.

A presença de artrose também foi um achado bastante significativo. Em dez pés (66\%) avaliados neste trabalho, houve progressão da artrose na MTF do hálux. Nenhuma articulação foi classificada como zero na radiografia obtida na avaliação final. Okuda et $a l^{(1)}$ demonstraram que, mesmo em intervalo de apenas três anos, a artrose na articulação MTF do hálux já pode estar presente, porém, às vezes, assintomática. Acreditamos que o tensionamento excessivo da cápsula na região da articulação MTF do hálux, a má redução dessa articulação após a cirurgia e lesões articulares prévias não diagnosticadas possam ser a causa do aparecimento e progressão radiográfica da artrose. Apesar de assintomática em alguns casos, a presença da artrose na MTF do hálux pode ser responsável pela perda do movimento observada no pós-operatório. Devemos lembrar que a osteotomia de base está indicada em casos de hálux valgo moderado a grave, que podem apresentar degeneração articular antes da correção cirúrgica. 
As osteotomias da base do primeiro metatarsal, ao corrigir o varismo do primeiro raio, provocam alterações no alinhamento, podendo ocorrer, além da elevação e/ou encurtamento, depressão, alongamento ou rotação ${ }^{(13)}$. O encurtamento e a elevação podem estar relacionados com a presença de lesão por transferência para os raios laterais (calosidades) ${ }^{(6-8)}$. A avaliação do encurtamento do I metatarsiano pode ser realizada a partir de várias fórmulas descritas na literatura ${ }^{(3,4,6)}$. No entanto, todas apresentam dificuldades quanto a sua reprodutibilidade, devido a fatores como a posição do pé ou da ampola do aparelho de raios $\mathrm{X}$ durante a realização do exame. Consideramos que o método por nós adotado minimiza essas variações, pois utiliza o II metatarsal como parâmetro.

Nyska et $a l^{(5)}$, em um estudo experimental com modelos ósseos, produziram uma grande variedade de osteotomias proximais no I metatarsal, observando mínima elevação e rotação em todas as técnicas utilizadas. Em outro trabalho ${ }^{(6)}$, os autores observaram encurtamento médio de $1,4 \mathrm{~mm}$ e elevação média de $2 \mathrm{~mm} ; 38 \%$ dos pés apresentaram elevação maior que $2 \mathrm{~mm}$, sem, no entanto, identificarem correlação estatisticamente significativa entre essas deformidades residuais e a ocorrência ou piora das lesões por transferência. Mann et $a l^{(16)}$ encontraram $28 \%$ de elevação do primeiro raio após osteotomias proximais cupuliformes, muito superior aos $9 \%$ encontrados por Zettl et $a l^{(9)}$, que consideraram a elevação uma limitação da técnica, que está associada à ocorrência de lesões por transferência. Trnka et $a l^{(8)}$, na avaliação de 42 pés, notaram encurtamento médio de $0,5 \mathrm{~cm}$ e desvio dorsal do primeiro metatarsal em 15 pés $(25 \%)$. Em nosso estudo, apesar de a elevação média de $0,52 \mathrm{~cm}$ e o encurtamento médio de $0,44 \mathrm{~cm}$ apresentarem significância do ponto de vista estatístico, não houve correlação estatística positiva entre calosidades plantares dolorosas e

\section{REFERÊNCIAS}

1. Okuda R, Kinoshita M, Morikawa J, Jotoku T, Abe M. Distal soft tissue procedure and proximal metatarsal osteotomy in hallux valgus. Clin Orthop Relat Res. 2000;(379):209-17.

2. Okuda R, Kinoshita M, Morikawa J, Jotoku T, Abe M. Proximal metatarsal osteotomy: relation between 1-to greater than 3-years results. Clin Orthop Relat Res. 2005;(435):191-6.

3. Coughlin MJ. Hallux Valgus. J Bone Joint Surg Am. 1996;78(6):932-66

4. Brodsky JW, Beischer AD, Robinson AH, Westra S, Negrine JP, Shabat S. Surgery for hallux valgus with proximal crescentic osteotomy causes variable postoperative pressure patterns. Clin Orthop Relat Res. 2006;(443):280-6.

5. Nyska M, Trnka HJ, Parks BG, Myerson MS: Proximal metatarsal osteotomies: A comparative geometric analysis conducted on sawbone models. Foot Ankle Int. 2002; 23(10):938-45.

6. Bozkurt M, Tigaran C, Dalstra M, Jensen NC, Linde F. Stability of a cannulated screw versus a Kirschne wire for the proximal crescentic osteotomy of the first metatarsal: a biomechanical study. J Foot Ankle Surg. 2004;43(3):138-43.

7. Veri JP, Pirani SP, Claridge R. Crescentic proximal osteotomy for moderate to severe hallux valgus: a mean 12,2 year follow-up study. Foot Ankle Int. 2001;22(10): 817-22.

8. Trnka HJ, Mühlbauer M, Zembsch A, Hungerford M, Ritschl P, Salzer M. Basal closing wedge osteotomy for correction of hallux valgus and metatarsus primus varus: 10 - to 22-year follow-up. Foot Ankle Int. 1999;20(3)171-7.

9. Zettl R, Trnka HJ, Easley M, Salzer M, Ritschl P. Moderate to severe hallux valgus lesões por transferência, com o encurtamento e elevação do primeiro metatarsal (calosidades foram observadas em quatro pés, sendo somente duas sintomáticas).

Veri et $a l^{(7)}$ notaram $5 \%$ de deformidades em varo, $11 \%$ de recidiva e $2 \%$ de novas lesões por transferências de carga. Além disso, observaram que a maior parte das novas queixas álgicas no antepé se localizavam na articulação MTF, sendo a maioria $(60 \%)$ relacionada com o hálux varo. Zettl et $a l^{(9)}$, em um estudo retrospectivo com 114 pés, observaram evolução para hálux varo em 9\% dos pés, dois pés desenvolveram hallux rigidu, e apenas uma recidiva. Trnka et $\mathrm{al}^{8}$ encontraram $17 \%$ de resultados clínicos ruins quanto à estética do primeiro raio, quando considerada a avaliação subjetiva do paciente. Quando relatam as complicações tardias, os mesmos autores enumeram $27 \%$ de deformidades em varo (16 em 60 pés), atribuindo essa deformidade a ressecção excessiva do joanete, correção excessiva do AIM I-II e a capsuloplastia com tensionamento excessivo. Apesar de não haver padronização nas cirurgias realizadas no grupo de pacientes avaliados neste trabalho (técnicas diferentes de osteotomia e de fixação), consideramos o índice de complicações tardias elevado, ou seja, nove dos 15 pés $(60 \%)$ com alguma complicação tardia, sendo quatro $(27 \%)$ deformidades em varo, três $(20 \%)$ recidivas e dois casos $(13 \%)$ com queixa de dor sem deformidade associada.

\section{CONCLUSÕES}

A técnica de osteotomia da base e realinhamento distal de partes moles, utilizada para correção de hálux valgo moderado a grave, mostrou elevado índice de complicações tardias. Devido ao elevado número dessas complicações, acreditamos que a eleição dessa técnica para correção do hálux valgo deve ser considerada com cautela.

deformity: correction with proximal crescentic osteotomy and distal soft tissue release. Arch Orthop Trauma Surg. 2000;120(7):397-402.

10. Kitaoka HB, Alexander IJ, Adelaar RS, Nunley JA, Myerson MS, Sanders M. Clinical rating systems for the ankle and hindfoot, midfoot, halux, and lesser toes. Foot Ankle Int. 1994;15(7):349-52.

11. Roukis TS, Jacobs PM, Dawson DM, Erdmann BB, Ringstrom JB. A prospective comparison of clinical, radiographic, and intraoperative features of hallux rigidus. $J$ Foot Ankle Surg. 2002;41(2):76-95.

12. Ignácio H, Chueire AG, Carvalho Filho G, Nascimento LV, Vasconcelos UMR, Barão GTF. Estudo retrospectivo da osteotomia de base do primeiro metatarsal como tratamento do hálux valgo. Acta Ortop Bras. 2006; 14(1):48-52.

13. Ruaro AF, Carvalho Junior AE, Fernandes TD, Salomão O, Aguilar JAG, Meyer AT. Estudo comparativo entre duas técnicas de osteotomia no tratamento do hálux valgo: análise clínica e radiográfica. Rev Bras Ortop. 2000;35(7):248-59.

14. Dreeben S, Mann RA: Advanced hallux valgus deformity. Long-term results utilizing the distal soft tissue procedure and proximal metatarsal osteotomy. FootAnkle Int. 1996;17(3):142-4.

15. Baykal B, Kirdemir V, Atesalp AS, Bek D, Tercan V. Modified crescentic metatarsal osteotomy and distal soft tissue procedures in hallux valgus. Mil Med. 2006; 171(12):1247-50.

16. Mann RA, Rudicel S, Graves SC. Repair of hallux valgus with a distal soft tissue procedure and proximal metatarsal osteotomy. A long-term follow-up. J Bone Joint Surg Am. 1992;74(1):124-9. 